\title{
New findings from old data: A semi- experimental value for the eQq of the nitrogen atom $\oplus$
}

Cite as: J. Chem. Phys. 153, 234307 (2020); https://doi.org/10.1063/5.0033071

Submitted: 13 October 2020 . Accepted: 30 November 2020 . Published Online: 21 December 2020

(D) Pablo Pinacho, (i) Daniel A. Obenchain, and (iD) Melanie Schnell

\section{COLLECTIONS}

EP This paper was selected as an Editor's Pick
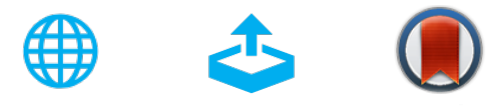

\section{ARTICLES YOU MAY BE INTERESTED IN}

Probing the deformation of [12]cycloparaphenylene molecular nanohoops adsorbed on metal surfaces by tip-enhanced Raman spectroscopy

The Journal of Chemical Physics 153, 244201 (2020); https://doi.org/10.1063/5.0033383

Machine learning with bond information for local structure optimizations in surface science The Journal of Chemical Physics 153, 234116 (2020); https://doi.org/10.1063/5.0033778

Directing excited state dynamics via chemical substitution: A systematic study of $\pi$-donors and $\pi$-acceptors at a carbon-carbon double bond

The Journal of Chemical Physics 153, 244307 (2020); https://doi.org/10.1063/5.0031689

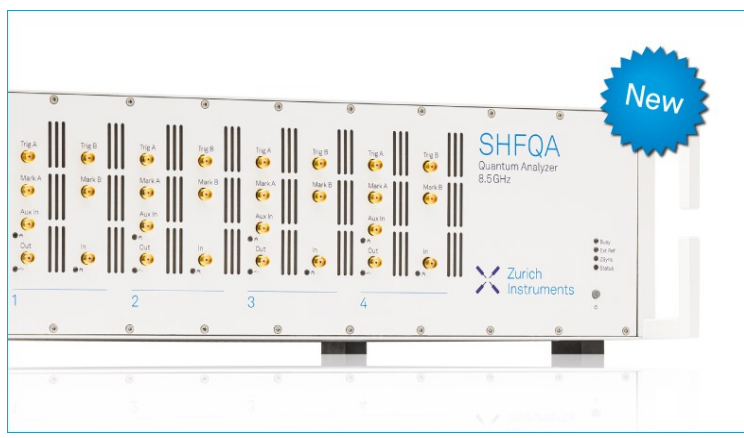

\section{Your Qubits. Measured.}

Meet the next generation of quantum analyzers

- Readout for up to 64 qubits

- Operation at up to $8.5 \mathrm{GHz}$

Find out more

- Signal optimization with minimal latency 


\title{
New findings from old data: A semi-experimental value for the eQq of the nitrogen atom (라
}

\author{
Cite as: J. Chem. Phys. 153, 234307 (2020); doi: 10.1063/5.0033071 \\ Submitted: 13 October 2020 - Accepted: 30 November 2020 • \\ Published Online: 21 December 2020
}

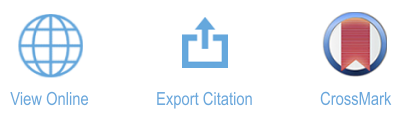

\author{
Pablo Pinacho, $^{1, a)}$ (D) Daniel A. Obenchain, ${ }^{1, b)}$ (D) and Melanie Schnell ${ }^{1,2, a)}$ (D)
}

\author{
AFFILIATIONS \\ 'Deutsches Elektronen-Synchrotron (DESY), Notkestraße 85, 22607 Hamburg, Germany \\ ${ }^{2}$ Institute of Physical Chemistry, Christian-Albrechts-Universität zu Kiel, Max-Eyth-Straße 1, 24118 Kiel, Germany
}

\begin{abstract}
a) Authors to whom correspondence should be addressed: pablo.pinacho@desy.de and melanie.schnell@desy.de
b) Present address: Institut für Physikalische Chemie, Universität Göttingen, Tammannstr. 6, 37077 Göttingen, Germany.
\end{abstract}

\begin{abstract}
Nuclear quadrupole coupling arises from the interaction of the nuclear quadrupole moment with the electric field gradient. Thus, it is associated with electron occupancy and the electronic structure of molecules. We demonstrate a simple method for planar molecules based on a direct correlation between the out-of-plane quadrupole coupling constant and the electron occupancy in the $p$ orbital perpendicular to the molecular plane. This method is applied to 98 molecular systems containing a ${ }^{14} \mathrm{~N}$ quadrupolar nucleus using data from more than 40 years of rotational spectroscopy and comparing the performance of three levels of theory from quantum-chemical computations. From this extensive dataset, we have analyzed chemical properties of molecules, such as the hybridization of the atom, and we could quantify the extent of polarization and resonance processes as well as physical characteristics of the quadrupolar nucleus, such as $e Q q$. This is a constant, which represents the interaction in the hypothetical case of having a single electron in an electronic orbital at the isolated nucleus, and its value has been under debate for a long time. Here, the $e Q q$ value has been determined for the ${ }^{14} \mathrm{~N}$ nucleus, and the methodology to calculate it for other nuclei is provided.
\end{abstract}

Published under license by AIP Publishing. https://doi.org/10.1063/5.0033071

\section{INTRODUCTION}

"Nuclear quadrupole coupling constants (NQCCs) in molecules depend on the nuclear quadrupole moments and the variation in electrostatic field at the nucleus." With this quote starts the canonical paper from Townes and Dailey about nuclear quadrupole effects and their relationship with the electronic structure of molecules. ${ }^{1}$ The Townes-Dailey (TD) model postulates that nuclear electric quadrupole coupling is caused by the interaction of the electric field gradient (EFG) from electrons in the valence shell $p$ orbitals (vide infra) with the nuclear quadrupole moment (Q) of atoms with a nuclear spin I $>1 / 2$. Therefore, the quadrupole interaction has an electronic origin, and the nuclear quadrupole coupling constants (NQCCs) in molecules can be related to the electronic structure of the molecule, chemical bonds, orbital hybridization of the quadrupolar nucleus, or resonance processes.

In order to obtain reliable information about the electronic structure or the chemical bonding, it is critical to understand how the NQCCs scale with the electronic charge. In fact, NQCCs are proportional to the number of unpaired electrons in the atomic orbitals related by a parameter ( $\chi_{0}$ or $e Q q_{\mathrm{n} 10}$, vide infra) that includes the elementary electric charge $e, Q$, and the $\operatorname{EFG}\left(q_{\mathrm{n} 10}\right) .^{1-3}$ An $e Q q_{\mathrm{n} 10}$ has a constant value for each quadrupolar nucleus since it represents the interaction due to a single unpaired electron in a $p$ orbital for the isolated nucleus. In $e Q q_{\mathrm{n} 10}$, the elementary charge $e$ is wellknown, ${ }^{5}$ the nuclear quadrupole moment $Q$ is specific for each nucleus and its values are tabulated, ${ }^{3}$ and $q_{\mathrm{n} 10}$ is the EFG, which can be computed from the molecular wave functions. In principle, it seems as all the components are known and deduction of electronic molecular properties from NQCCs is a simple process. In reality, though, this is a more challenging task; computing the EFG is complicated since it requires considering the contribution of all the charges, and only an approximation to the wave functions can be computed. Approximations used to speed up calculations, like pseudopotentials, sacrifice accuracy in predicting the EFG. This is particularly true for late periodic table elements such 
as iodine and gold. ${ }^{6,7}$ As a result, some $e Q q_{\mathrm{n} 10}$ values seem to be not reliable, or different values have been reported with large discrepancies between them. For example, for ${ }^{127} \mathrm{I}, e Q q_{510}$ was tabulated as $292.71 \mathrm{MHz},{ }^{3}$ but it was mistyped, with the actual value being $2292.71 \mathrm{MHz} .{ }^{8}$ In the case of ${ }^{14} \mathrm{~N}$, there is no agreement on its value to date. First estimations of $e Q q_{210}\left({ }^{14} \mathrm{~N}\right)$ ranged from $-10 \mathrm{MHz}$ to $-24 \mathrm{MHz}$. This value was later limited to be between $-10 \mathrm{MHz}$ and $-11 \mathrm{MHz} .^{9-11}$ A more modern update by quantumbeat and radio-frequency resonance spectroscopy reported it to be $11.2 \pm 0.2 \mathrm{MHz}$ for the ${ }^{14} \mathrm{~N}$ atom. ${ }^{12}$ Most of the contemporary authors take the negative sign from the first data, with the updated value of $11.2 \mathrm{MHz}$.

The analysis of the hyperfine structure using different spectroscopic techniques can provide information about the nuclear quadrupole interaction, the electronic structure, and chemical properties. Many molecules owning quadrupolar nuclei can be studied by nuclear magnetic resonance $(\mathrm{NMR})^{13,14}$ or by rotational spectroscopy. Both techniques have much in common ${ }^{15}$ but they differ in the state of the matter of the molecules investigated. Classical NMR experiments can be performed in liquid samples, and solid state NMR is a powerful technique for chemical analysis, especially for quadrupolar nuclei. ${ }^{14,16}$ On the other hand, rotational spectroscopy studies gas phase molecules and molecular clusters generated in a molecular beam, creating a molecular environment free from external interactions, such as crystal packing forces in solids. In rotational spectroscopy, the nuclear quadrupole coupling interaction is observed as a hyperfine structure of the rotational transitions in the spectra. Rotational spectrometers are characterized as high resolution, which involves the measurement of accurate frequencies for the rotational and hyperfine transitions. This is translated into an accurate determination of NQCCs. This high accuracy and the possibility to study molecules and clusters in the gas phase make rotational spectroscopy an excellent technique to investigate intrinsic atomic and molecular properties.

In this work, we have focused on ${ }^{14} \mathrm{~N}$-containing molecules studied by rotational spectroscopy to explore the relationship between the molecular structure, NQCCs, and $e Q q_{\mathrm{n} 10}$, aiming at information about molecular properties and at deriving an accurate, reliable $e Q q_{210}$ value for the ${ }^{14} \mathrm{~N}$ atom from experimental data.

\section{THEORETICAL BACKGROUND}

In the Townes-Dailey (TD) model, NQCCs $\left(\chi_{\mathrm{ij}}, i, j=x, y, z\right)$ arise from the interaction of the nuclear quadrupole moment $(Q)$ for those nuclei with a non-spherical distribution of the charge, i.e., with a nuclear spin $\mathrm{I}>1 / 2$, with the $\operatorname{EFG}\left(q_{\mathrm{ij}}\right)$ at the nucleus $\left(\chi_{\mathrm{ij}}=e Q q_{\mathrm{ij}}\right)$. To estimate $q_{\mathrm{ij}}$, it would be needed, in principle, to consider the EFG due to the charges in the molecule $\left(q_{\mathrm{mol}}\right)$, but the TD model considers some approximations to simplify the calculation. ${ }^{2,3}$ EFG scales with $1 / \mathrm{r}^{3}$, thus, only the electrons of the coupling nucleus have a significant influence $\left(q_{\mathrm{mol}}=q_{\mathrm{nlm}}\right)$. The electrons in the core filled shells and those in the valence shell $s$ orbitals are spherically symmetric, and their contribution can be neglected. The contribution of $d$ electrons has been estimated to be lower than those in a $p$ orbital by a factor of 47 and is thus omitted here. ${ }^{8}$ In the spacefixed axis system, the maximum effective value for the electric field gradient occurs for the quantum numbers $l=1$ and $m_{1}=0$. Thus, the electric field gradient that produces nuclear quadrupole coupling can be approximately attributed to an uneven occupancy of the valence shell $p$ orbitals of the coupling atom $\left.\left(q_{\mathrm{nlm}}=q_{\mathrm{n} 10}\right)\right)^{2,3}$ With these approximations, the EFG caused by a single electron is

$$
\begin{aligned}
& q_{x}=-\left(\frac{n_{y}+n_{z}}{2}-n_{x}\right) q_{n 10}, \\
& q_{y}=-\left(\frac{n_{z}+n_{x}}{2}-n_{y}\right) q_{n 10}, \\
& q_{z}=-\left(\frac{n_{x}+n_{y}}{2}-n_{z}\right) q_{n 10} .
\end{aligned}
$$

The term inside brackets represents the unequal occupancy of the $p$ orbitals with $n_{\mathrm{i}}(i=x, y$ or $z)$ being the number of electrons in the $p_{\mathrm{i}}$ orbital, which can be computed, for example, from Natural Bond Orbital (NBO) calculations. ${ }^{17,18}$ This term is more frequently represented as $-\left(U_{\mathrm{p}}\right)_{\mathrm{i}}(i=x, y$ or $z)$ and corresponds to the electron density deficit or excess along the $i$ axis at the coupling nucleus. If the population of the $p_{z}$ orbital is greater than the semi-sum of the population of $p_{\mathrm{x}}$ and $p_{\mathrm{y}}\left[n_{z}>\left(\frac{n_{x}+n_{y}}{2}\right)\right]$, then $-\left(U_{\mathrm{p}}\right)_{\mathrm{z}}$ will be positive, indicating an excess of electron density along the $z$ axis. On the other hand, if $n_{z}<\left(\frac{n_{x}+n_{y}}{2}\right),-\left(U_{\mathrm{p}}\right)_{\mathrm{z}}$ will be negative, indicating a deficit of electron density along the $z$ axis.

The original TD model has been extended ${ }^{8}$ and modified $^{19}$ to improve its performance. Recently, Rinald and Wu have introduced the concept of valence $p$-orbital population anisotropy (VPPA) in their modified Townes-Dailey model, showing how the unequal occupancy of $p$-orbitals is related to the NQC tensor and the $\chi_{0}$ constant. Their VPPA term is equivalent to our $-\left(U_{\mathrm{p}}\right)_{\mathrm{z}}$ since both illustrate the unbalanced (or unpaired) number of electrons in a $p$ orbital, based on the electronic populations from natural molecular orbital calculations. We will continue using $-\left(U_{\mathrm{p}}\right)_{\mathrm{z}}$ and $e Q q_{210}$ considering that it is the classical nomenclature defined by Townes and Dailey, and most of the researchers in rotational spectroscopy may be familiar with this terminology.

Substituting $q_{\mathrm{z}}$ from Eq. (3) with $\chi_{\mathrm{ij}}=e Q q_{\mathrm{ij}}$ gives

$$
\begin{gathered}
\chi_{z z}=e Q\left(-\left(\frac{n_{x}+n_{y}}{2}-n_{z}\right) q_{n 10}\right)=e Q\left(-\left(U_{p}\right)_{z} q_{n 10}\right), \\
\chi_{z z} / e Q q_{n 10}=-\left(U_{p}\right)_{z} .
\end{gathered}
$$

From these equations, it is evident how sensitive the NQCC values are to the electronic environment, making them unique for each molecule, isomer, and conformer. ${ }^{2,20,21}$ Experimentally, the measurable NQCCs are defined in the molecular inertial axis system, $\chi_{\alpha \beta}(\alpha, \beta=a, b$ or $c)$, but $e Q q_{\mathrm{n} 10}$ is directly related to the NQCCs in the axis system of the quadrupolar atom $\left(\chi_{i j}\right)$. Transformation between both axis systems will require the diagonalization of the quadrupole coupling tensor, which can only be performed if the tensor is completely known. For certain nuclei, which are strongly coupled and thus show rather large quadrupole coupling splitting, such as bromine and iodine, it is usually possible to determine the 

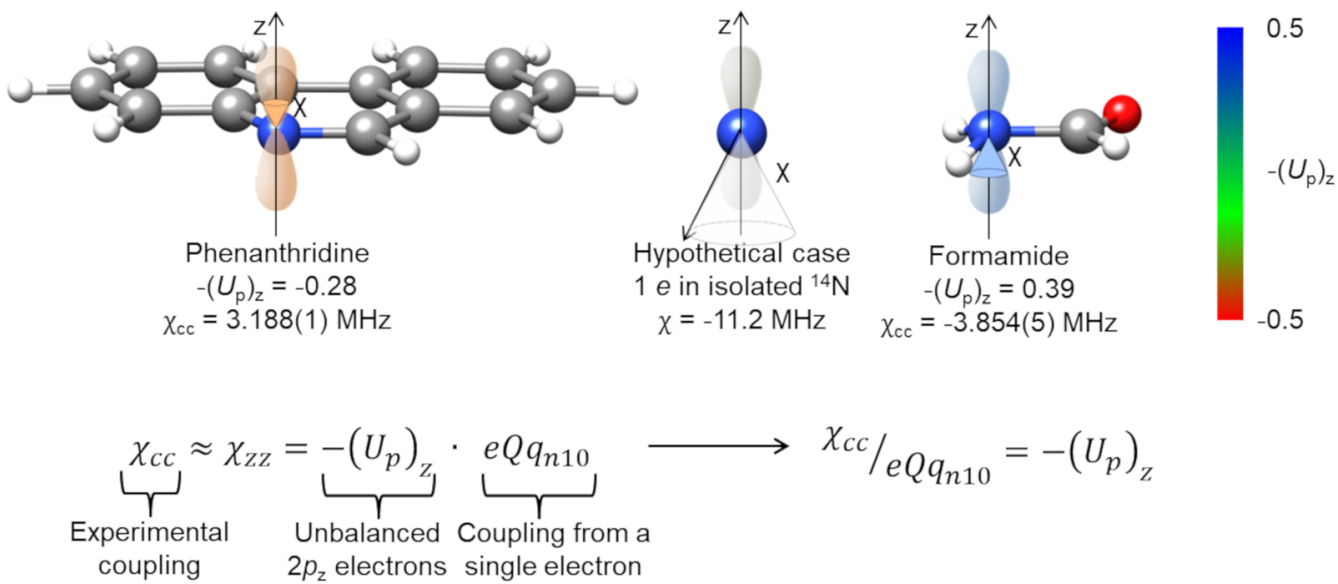

FIG. 1. The approximation employed in this work and the correlation between $\chi_{\mathrm{cc}},-\left(U_{\mathrm{p}}\right)_{\mathrm{z}}$, and $e Q q_{\mathrm{n} 10}$. Representation of the quadrupole coupling in phenanthridine and formamide based on their $-\left(U_{p}\right)_{z}$ and the hypothetical case of an electron in an isolated ${ }^{14} \mathrm{~N}$ nucleus considering $e Q q_{210}$ as $-11.2 \mathrm{MHz}$.

whole tensor experimentally. For the weaker coupling nuclei, such as ${ }^{14} \mathrm{~N}$, the complete NQC tensor can often not be determined since the effect of the off-diagonal elements is almost negligible or the intensity of the transitions is below the detection limits of the instrumentation. Missing terms can be taken from quantum-chemical calculations to complete the experimental tensor, or in another approximation, the theoretical tensor can be diagonalized and then the Cartesian transformation matrix employed is applied to the experimental values to obtain approximated $\chi_{i j}$ values. Nevertheless, for certain molecular systems, this process may not be needed. Provided the planarity or almost planarity of a molecular system, the perpendicular NQCC will be parallel to one of the $\chi_{\text {ii }}$ constants, and from Eq. (5), it can be correlated with the corresponding $-\left(U_{\mathrm{p}}\right)_{\mathrm{i}}$. The association between $\chi_{\alpha \beta}$ and $\chi_{\mathrm{ij}}$ is not always straightforward since there are several ways of correlating the molecular axis system with the quadrupolar nucleus axis system. ${ }^{3}$ The NBO calculations performed in this work indicate that for all the molecules studied, the $2 p_{z}$ orbital is perpendicular to the molecular plane. Based on that, we have considered here that the NQCC perpendicular to the molecular plane, $\chi_{\mathrm{cc}}$, is approximately equivalent to $\chi_{\mathrm{zz}}$. In this case, it can be correlated with $-\left(U_{\mathrm{p}}\right)_{\mathrm{z}}$, which represents the unbalanced electronic charge from the perpendicular $2 p_{z}$ orbital (Fig. 1). This approximation has been used in other studies, ${ }^{22-24}$ where relevant information was concluded from the evolution of $\chi_{\mathrm{cc}}$ for a series of complexes, and it is extended to a variety of molecules here.

The aim of this work is to provide a proof of principle of this approach and to apply it to an extensive dataset of the available molecules in the bibliography. This method could be extended later to more molecular systems and other quadrupolar nuclei.

\section{METHODOLOGY}

A total of 105 data points for ${ }^{14} \mathrm{~N}$ NQCCs have been used for this study. They have been obtained from a bibliographical search of a plethora of studies covering more than 40 years of rotational spectroscopy from many research groups devoted to Fourier transform microwave (FTMW) spectroscopy. Different experimental setups based on this technique were used: Fabry-Perot cavity FTMW spectrometers ${ }^{25}$ or broadband chirped-pulse FTMW instruments. ${ }^{26}$ The bibliographical search for experimental data points was guided by the collection of molecules at Professor Bailey's website. ${ }^{27}$ Table S1 in the supplementary material shows the experimental rotational and quadrupole coupling constants together with $P_{\mathrm{cc}}$ and $\chi_{\mathrm{cc}} / e Q q_{210}$. The approximation employed $\left[\chi_{\mathrm{cc}} \approx \chi_{\mathrm{zz}}\right.$ $\left.=-\left(U_{\mathrm{p}}\right)_{\mathrm{z}} \cdot e Q q_{\mathrm{n} 10}\right]$ is only valid for planar systems. The planarity of a molecule or complex can be evaluated by the $P_{\mathrm{cc}}$ parameter, which gives the mass extension out of the molecular plane; the greater the $P_{c c}$, the less planar the system. We have observed that even for complexes with a planar moment of up to $15 \mu \AA^{2}$, such as the complexes with three water molecules with either ethyl carbamate or phenanthridine (PAN) (Table S1), the approximation is still valid. The complete list of references for the experimental values is also given in the supplementary material.

The calculations to obtain $-\left(U_{\mathrm{p}}\right)_{\mathrm{z}}$ were performed at three levels of theory: $\mathrm{B} 3 \mathrm{LYP}^{28} / \mathrm{cc}-\mathrm{pVDZ},{ }^{29} \mathrm{~B} 3 \mathrm{PW} 91^{14,30} / 6-311+\mathrm{G}(\mathrm{df}, \mathrm{pd}),{ }^{31}$ and $\mathrm{MP}^{32} / \mathrm{cc}-\mathrm{pVDZ}$. B3LYP has been chosen based on its popularity and relatively low computational cost, ${ }^{33}$ B3PW91/6$311+\mathrm{G}(\mathrm{df}, \mathrm{pd})$ has been reported to be a specially good level of theory to describe ${ }^{14} \mathrm{~N}$ quadrupolar momentum, ${ }^{34}$ and MP2 was chosen to provide a comparison to the density functional theory (DFT) values. In some sections of this study, only the results at the B3PW91/6-311+G(df,pd) level of theory are given since it is the recommended method, ${ }^{34}$ but the results from the three levels of theory are comparable. The first step was to perform structure optimizations using the Gaussian suite; ${ }^{35}$ after that, we performed NBO calculations using the optimized geometry to obtain the electron occupancy of the valence-shell orbitals. The accuracy of the quantum-chemical calculations was tested by comparison of the predicted rotational constants and NQCCs with the experimental data given in the bibliography. For every level of theory, there are some 
data points that do not reproduce the experimental information or for which the calculation did not converge into a stable structure. These data points were excluded from the analysis for their tendency line (vide infra). Tables S2-S4 show the predicted parameters for the three levels of theory employed indicating which data points were excluded.

\section{RESULTS AND DISCUSSION}

To corroborate the validity of Eq. (5), we compared the experimental $\chi_{\alpha \alpha} / e Q q_{210}(\alpha=a, b$ or $c)$ vs the calculated $-\left(U_{\mathrm{p}}\right)_{\mathrm{i}}(i=x, y$ or $z)$ at the three levels of theory for 105 data points of ${ }^{14} \mathrm{~N}$. At this stage, $e Q q_{210}$ was taken as $-11.2 \mathrm{MHz},{ }^{8,12}$ which is a good starting value despite the inconsistencies of previous studies. There are nine possible $-\left(U_{\mathrm{p}}\right)_{\mathrm{i}}$ vs $\chi_{\alpha \alpha}$ combinations, which have been represented in the matrix arrangement for the B3PW91/6-311+G(df,pd) level of theory (Fig. 2). The strong correlation between $-\left(U_{\mathrm{p}}\right)_{\mathrm{z}}$ and $\chi_{\mathrm{cc}}$ confirms that the approximation $\chi_{\mathrm{cc}} \approx \chi_{\mathrm{zz}}=-\left(U_{\mathrm{p}}\right)_{\mathrm{z}} \cdot e Q q_{210}$ is valid for the molecular systems chosen. A linear feature but with higher dispersion of the data seems to appear for the pairs, $-\left(U_{\mathrm{p}}\right)_{\mathrm{x}} / \chi_{\mathrm{aa}}$ and $-\left(U_{\mathrm{p}}\right)_{\mathrm{y}} / \chi_{\mathrm{bb}}$, although it could be expected to follow a higher correlation degree after the diagonalization of the quadrupole tensor.
In Fig. 3, the experimental $\chi_{\mathrm{cc}} / e Q q_{210}$ is compared with the calculated $-\left(U_{\mathrm{p}}\right)_{\mathrm{z}}$ at the three levels of theory, showing that the three quantum-chemical methods give comparable results. Some systematic effects can be observed, i.e., the theoretical NQCC values are predicted to be larger than the experimental ones for the included aniline-like molecules (aniline, methylaniline, dimethylaniline, 4-aminobenzonitrile, and 4,4'-dimethylaminobenzonitrile; see Tables S2-S4 in the supplementary material).

The values do not cover the complete line, but are concentrated between the absolute values $(0.45-0.2)$ with just a few examples out of this range and close to 0 . Since the values are related with the electron occupancy of the nitrogen $2 s$ and $2 p$ orbitals, a possible explanation could be that only certain values of the electron occupancy might give rise to stable molecules. The molecules and complexes studied cover the different hybridization possibilities for the nitrogen atom, $s p, s p^{2}$, and $s p^{3}$, and they can be classified according to their position (Fig. 4). The negative quadrant (negative value for $\chi_{\mathrm{cc}} / e Q q_{210}$; positive $\chi_{\mathrm{cc}}$ ) contains molecules with the $\mathrm{N}$ in the $s p$ configuration and those with the $s p^{2}$ hybridization in which the lone pair of nitrogen is localized in one of the $s p^{2}$ orbitals and the remaining non-hybridized $2 p$ orbital is involved in a resonance process. Examples of molecules in this quadrant are pyridine, oxazole, benzonitrile, and molecules with
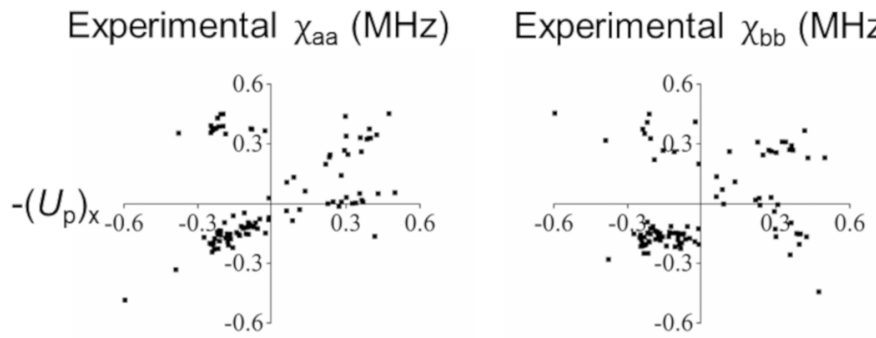

Experimental $\chi_{\mathrm{cc}}(\mathrm{MHz})$
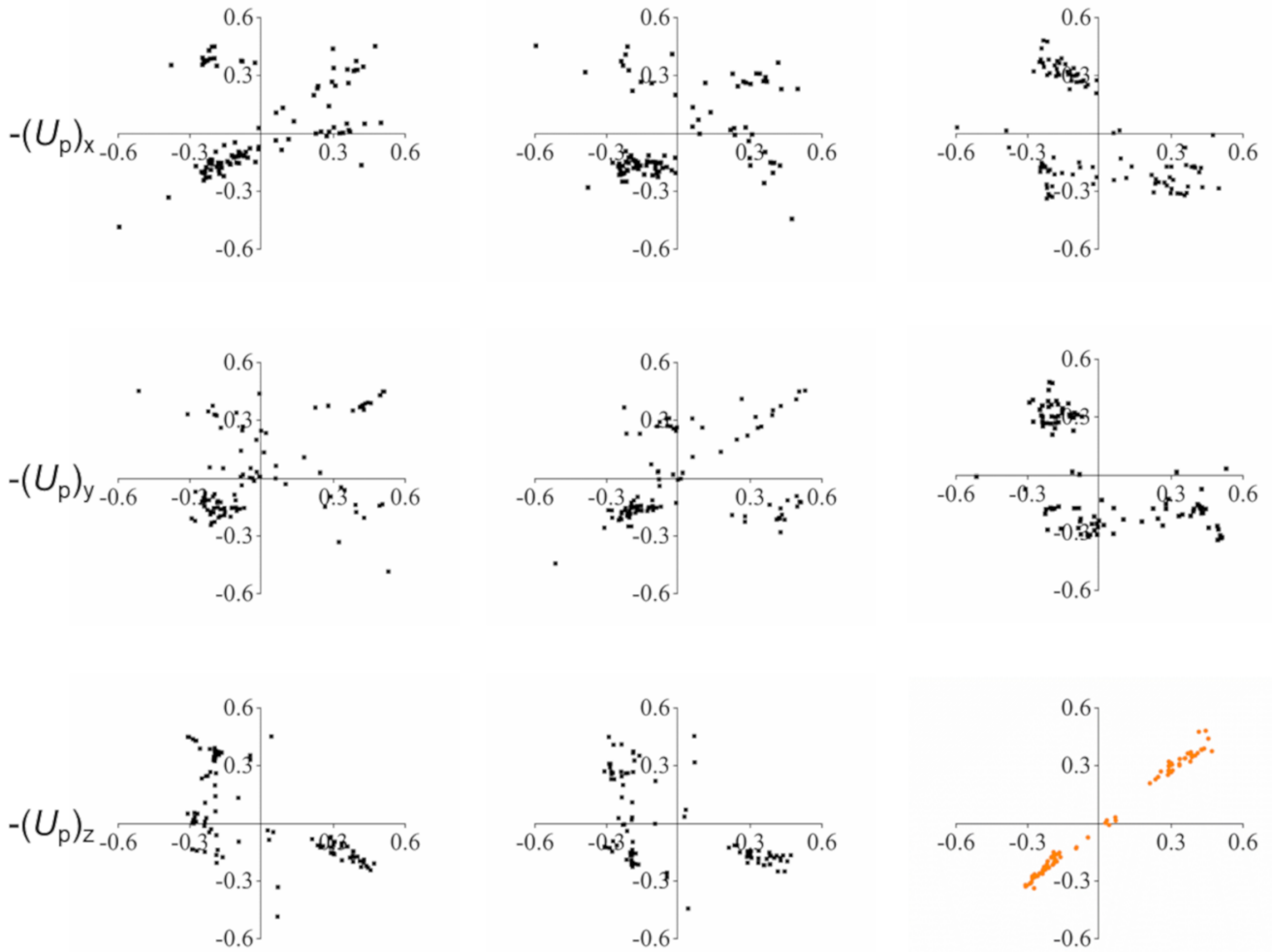

FIG. 2. The nine possible combinations of experimental $\chi_{\alpha a}(\alpha=a, b$ or $c)$ vs $-\left(U_{p}\right)_{i}(i=x, y$ or $z)$ in the matrix arrangement. All the experimental quadrupole values are divided by $\mathrm{e} Q q_{210}$ taken as $-11.2 \mathrm{MHz}$. 


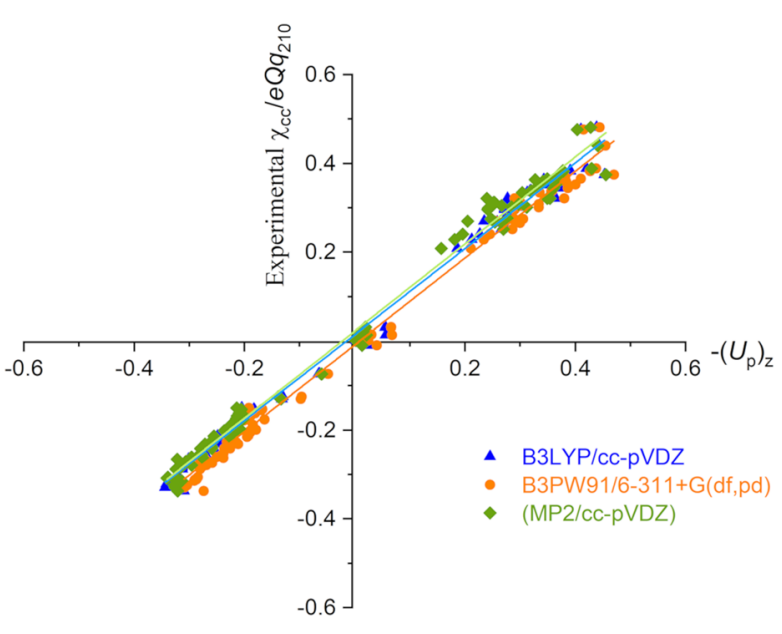

FIG. 3. Experimental $\chi_{c c} / e Q q_{210}$ vs calculated $-\left(U_{p}\right)_{z}$ at the B3LYP/cc-pVDZ, B3PW91/6-311+G(df,pd), and MP2/cc-pVDZ levels of theory. A value of $e Q q_{210}$ $=-11.2 \mathrm{MHz}$ was used.

similar chemical environments. The molecules with values close to 0 are 3-methylisoxazole, 4-methylisoxazole, 5-methylisoxazole, isocyanobenzene, and pentafluoroisocyanobenzene. For these molecules, the nitrogen atom has a similar hybridization to the molecules in the negative quadrant, but it is attached to a highly electronegative group. The positive quadrant (positive $\chi_{\mathrm{cc}} / e Q q_{210}$; negative $\chi_{\mathrm{cc}}$ ) includes molecules with $s p^{3}$ hybridization, and molecules with $s p^{2}$ hybridization in which the lone pair from the nitrogen atom is localized in the non-hybridized $2 p$ orbital. Data points in this section belong to amines, amides, and pyrrole- or indole-like molecules. There are also examples of molecules with two nitrogen atoms with different hybridization schemes, such

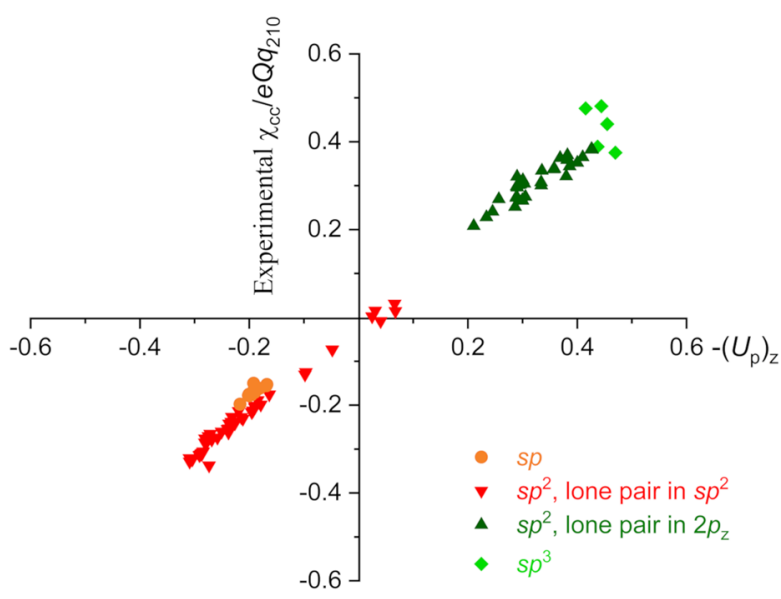

FIG. 4. Experimental $\chi_{c c} / e Q q_{210}$ vs calculated $-\left(U_{p}\right)_{z}$ at the B3PW91/6$311+G(d f, p d)$ level of theory illustrating different hybridizations for the nitrogen atom in the different quadrants. A value of $e Q q_{210}=-11.2 \mathrm{MHz}$ was used. us imidazole or 4-aminobenzonitrile. Another, simpler classification is possible. Molecules in the negative quadrant or close to 0 belong to data points in which the nitrogen is not attached to a hydrogen atom, while molecules in the positive quadrant have a $\mathrm{N}-\mathrm{H}$ bond.

\section{A. Polarization and resonance effects in complexes}

From the definition of $-\left(U_{\mathrm{p}}\right)_{\mathrm{z}}$ given above, a negative value as in phenanthridine (PAN) corresponds to an electron density deficit at the ${ }^{14} \mathrm{~N}$ nucleus along the $z$ axis, while a positive value as in formamide indicates an excess of electron density. ${ }^{3}$ For both cases, the monomer and complexes with up to three molecules of water have been measured, ${ }^{22-24,36}$ and the values of $\chi_{\mathrm{cc}}$ show a steady tendency (either an increase or a decrease) with the hydration degree (Fig. 5). The change in $\chi_{\mathrm{cc}}$ can be associated with the electronic environment at the ${ }^{14} \mathrm{~N}$ nucleus and therefore with the polarization via resonance processes. In the series of microsolvated complexes of PAN, $\chi_{\mathrm{cc}} / e Q q_{210}$ increases with the number of water molecules, which corresponds to an increase in the electron density along the $z$ axis at the nitrogen nucleus. ${ }^{24}$ In the series of microsolvated complexes of formamide, $\chi_{\mathrm{cc}}$ increases as more molecules of water are added, and consequently, as a result of the negative sign of $e Q q_{210} ; \chi_{\mathrm{cc}} / e Q q_{210}$ decreases. This illustrates the decrease in the electron density excess with the cluster size (Fig. 5). ${ }^{22,23}$ The tendency of $\chi_{\mathrm{cc}}$ for both systems is in good agreement with the polarization expected from the resonance forms that can be deduced from the electronic delocalization. In PAN, the resonance of the $\pi$-system creates a partial negative charge at the nitrogen nucleus (Fig. S1). The resonance can be extended to the water molecules, and therefore as the cluster increases its size, there is more resonance and the electron density at the nitrogen atom increases. In formamide, resonance induces a partial positive charge at the nitrogen atom (Fig. S1). As the cluster size increases, the electron

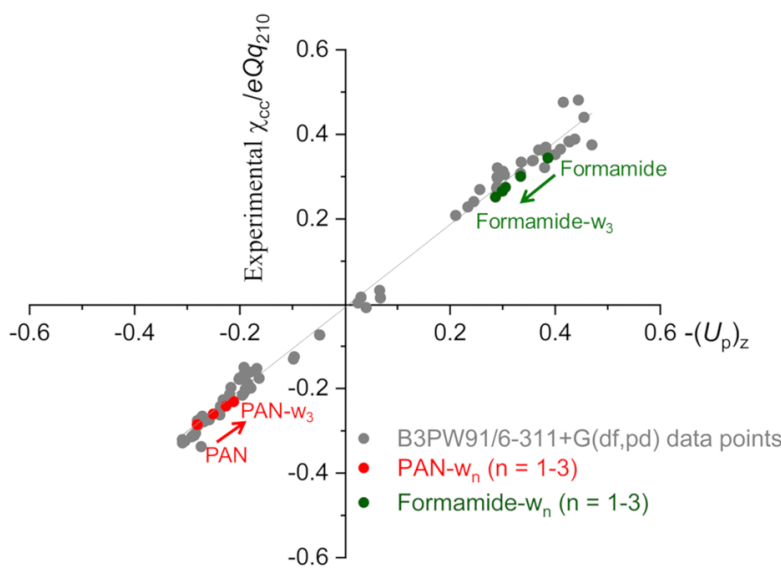

FIG. 5. Experimental $\chi_{c c} / e Q q_{210}$ vs $-\left(U_{p}\right)_{z}$ at $B 3 P W 91 / 6-311+G(d f, p d)$. The data points corresponding to complexes with up to three molecules of water for phenanthridine $\left(\mathrm{PAN}-\mathrm{w}_{\mathrm{n}}\right)$ and formamide- $\mathrm{w}_{n}$ have been highlighted in red and green, respectively. The changes in $\chi_{c c} / e Q q_{210}$ and $-\left(U_{p}\right)_{z}$ from the monomer to complexes with increasing water molecules are represented by the arrows. $A$ value of $e Q q_{210}=-11.2 \mathrm{MHz}$ was used. 
density at the nitrogen atom decreases. The experimental values of $\chi_{\mathrm{cc}}$ reproduce perfectly the evolution of the electron density on both molecular systems (Fig. 5). Similar tendencies in $\chi_{\mathrm{cc}}$ have been observed also for monomers and complexes with water of pyridine, benzonitrile, indole, pyrrole, azetidinone, and ethyl carbamate (Fig. S2). Although the dataset is more limited, the change in $\chi_{\mathrm{cc}}$ agrees with the expected behavior according to their resonance forms.

\section{B. Electron density and resonance effects in aromatic rings}

Resonance can be evidenced by trends in the NQCCs not only in series of complexes, but also in molecules with different substituents. In aromatic rings, the influence of substituents on the electron density of the ring is well known. The substituents can have two effects, inductive or mesomeric, and both can be either positive (electron donating) or negative (electron withdrawing). Depending on the atom and its relative position, the substituent affects the electron density of the respective atoms in the ring differently and in turn affects the molecular structure. This is an important principle for synthetic chemistry, and the substituents and their effects are well characterized. The quantification of the effects of electron-donating or electron-withdrawing groups on molecular structures is more challenging. Several studies have reported the observation of structural changes by means of microwave spectroscopy in aromatic rings. ${ }^{37-42}$ Experimentally, the determination of molecular structures relies on the observation of every possible mono-isotopic substitution, either in natural abundance or using enriched samples, which can be a challenge. The trends in the values of the NQCC $\chi_{\mathrm{cc}}$ of ${ }^{14} \mathrm{~N}$ in heteroaromatic rings can provide further confirmation of resonance and allow us to trace back the electron density even if the molecular structures are not determined.

In the following text, the approach will be exemplified for pyridine (pyr) and fluoro-substituted pyridines (F-pyr). ${ }^{39,40}$ Fluorine is a common substituent of aromatic rings and induces inductive and mesomeric effects, but with different sign. It extracts electronic charge from the ring since it is more electronegative than the carbon atoms in the aromatic ring. In addition, it donates electronic charge through resonance of the lone pairs (see Fig. S3 for resonance structures). The consequence on the electronic charge results from the addition of both effects and depends on the position of the fluorine substituent in the ring. The relationship between the experimental $\chi_{\mathrm{cc}} / e Q q_{210}$ and the calculated $-\left(U_{\mathrm{p}}\right)_{\mathrm{z}}$ at the B3PW91/6$311+\mathrm{G}(\mathrm{df}, \mathrm{pd})$ level of theory of the pyr and F-pyr molecules follows the described tendency line (see Figs. 6 and S4 for a zoom-in). Fluorine substitution in different positions with respect to the nitrogen atom affects the electron density at the ${ }^{14} \mathrm{~N}$ atom differently, resulting in changes of the $\chi_{\mathrm{cc}}$ values. The experimental values of the NQCC can be used as a probe to quantify the amount of electron density delocalization in molecules with one or also multiple substituents, for which the effect adds up.

Table I shows experimental $\chi_{\mathrm{cc}}$, the calculated $2 p_{\mathrm{z}}$ electron occupancy, and $-\left(U_{\mathrm{p}}\right)_{\mathrm{z}}$ computed at the B3PW91/6-311+G(df,pd) level of theory. Table S5 shows the values at the three levels of theory. The effect of fluorine substitution in the three possible positions has been calculated from the mono-substituted molecules, and

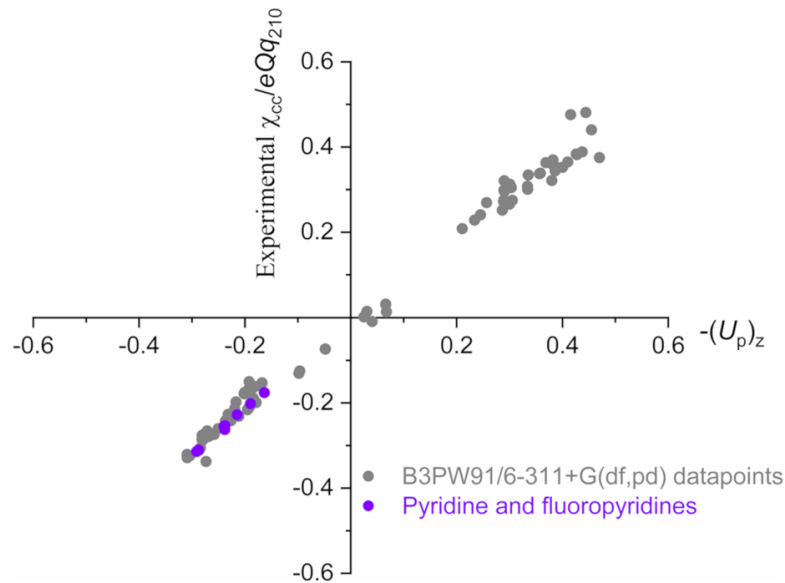

FIG. 6. Experimental $\chi_{c c} / e Q q_{210}$ vs $-\left(U_{p}\right)_{z}$ at $B 3 P W 91 / 6-311+G(d f, p d)$. The data points corresponding to pyridine and fluoropyridines have been highlighted in purple. A value of $e Q q_{210}=-11.2 \mathrm{MHz}$ was used.

then these values have been used to extrapolate the same properties for difluoro- and pentafluoropyridine. The extrapolated values for experimental $\chi_{\mathrm{cc}}, 2 p_{\mathrm{z}}$ electron occupancy, and $-\left(U_{\mathrm{p}}\right)_{\mathrm{z}}$ match with the experimental ones. The net effect of fluorine in the 2-position (ortho-) will be to donate 0.04 electrons to the $2 p_{z}$ orbital, affecting the $\chi_{\mathrm{cc}}$ value by $-0.638 \mathrm{MHz}$. This change is preserved in every system with a fluorine atom in the 2-position, even if more fluorine atoms are present. In 4- (para-) and 3- (meta-) positions, fluorine will donate 0.02 or extract around 0.007 electrons to the $2 p_{\mathrm{z}}$ orbital, respectively. The comparison between the extrapolated and the experimental $\chi_{\mathrm{cc}}$ allows tracing back the electron density and illustrates the electron donating or withdrawing processes. The change in the electronic population of the $2 p_{z}$ orbital could be in principle derived from the Extended Townes-Dailey (ETD) analysis, ${ }^{8,43}$ which also requires some approximations and assumptions based on quantum-chemical calculations. The application of the ETD is out of the scope of this work but similar conclusions could be expected.

\section{C. $e Q q$}

Besides the validation of the method and obtaining information about electron density through the experimental NQCC, one important aim of this work is the derivation of a semi-experimental value for the $e Q q_{210}$ parameter for ${ }^{14} \mathrm{~N}$. In previous sections, we have assumed $e Q q_{210}=-11.2 \mathrm{MHz},{ }^{8}$ but we can also determine this critical parameter from the selected dataset. If we represent directly the experimental values of $\chi_{\mathrm{cc}}$ instead of $\chi_{\mathrm{cc}} / e Q q_{210}$ vs $-\left(U_{\mathrm{p}}\right)_{\mathrm{z}}$, the gradient of the line should be $e Q q_{210}$ (Fig. 7). To obtain the value of the gradient, we performed a linear fit of the data points constraining the line to pass through 0 . The experimental regression line does not pass exactly through the 0.0 point (Fig. 7 ), but in the fit, we set that constraint assuming that no unbalanced $2 p_{z}$ electrons would correspond to $0 \chi_{\mathrm{cc}}$. We performed the fit for the calculated values of $-\left(U_{\mathrm{p}}\right)_{\mathrm{z}}$ at the three levels of theory and compared the results. 
TABLE I. Experimental $\chi_{c c}$, predicted and extrapolated $2 p_{z}$ electron occupancy, and $-\left(U_{p}\right)_{z}$ at B3PW91/6-311 $+G(d f, p d)$ for pyridine, monofluoropyridines, difluoropyridines, and pentafluoropyridine.

\begin{tabular}{|c|c|c|c|c|c|c|c|c|c|}
\hline & \multirow{2}{*}{$\begin{array}{c}\text { Experimental } \\
\chi_{\mathrm{cc}}(\mathrm{MHz})\end{array}$} & \multicolumn{2}{|c|}{ Theoretical (B3PW91) } & \multicolumn{3}{|c|}{ Fluorine effect } & \multicolumn{3}{|c|}{ Extrapolated fluorine effect } \\
\hline & & $2 p_{\mathrm{z}}$ & $-\left(U_{\mathrm{p}}\right)_{\mathrm{z}}$ & $\Delta \chi_{\mathrm{cc}}(\mathrm{MHz})$ & $\Delta 2 p_{\mathrm{z}}$ & $\Delta\left[-\left(U_{\mathrm{p}}\right)_{\mathrm{z}}\right]$ & $\chi_{\mathrm{cc}}(\mathrm{MHz})$ & $2 p_{\mathrm{z}}$ & $-\left(U_{\mathrm{p}}\right)_{\mathrm{z}}$ \\
\hline Pyridine & 3.474 & 1.160 & -0.288 & & $\ldots$ & $\ldots$ & $\ldots$ & $\ldots$ & $\ldots$ \\
\hline 2-fluoropyridine & 2.837 & 1.203 & -0.239 & $-0.638^{\mathrm{a}}$ & 0.043 & 0.049 & $\ldots$ & $\ldots$ & $\ldots$ \\
\hline 3-fluoropyridine & 3.501 & 1.153 & -0.290 & 0.027 & -0.007 & -0.002 & $\ldots$ & $\ldots$ & $\ldots$ \\
\hline 4-fluoropyridine & $2.997^{\mathrm{b}}$ & 1.179 & -0.265 & -0.477 & 0.019 & 0.023 & $\ldots$ & $\ldots$ & $\ldots$ \\
\hline 2,3-difluoropyridine & 2.940 & 1.199 & -0.238 & $\ldots$ & $\ldots$ & $\ldots$ & $2.863^{\mathrm{c}}$ & 1.196 & -0.240 \\
\hline 2,4-difluoropyridine & 2.548 & 1.222 & -0.215 & $\ldots$ & $\ldots$ & $\ldots$ & 2.360 & 1.223 & -0.215 \\
\hline 2,5-difluoropyridine & 2.851 & 1.197 & -0.240 & $\ldots$ & $\ldots$ & $\ldots$ & 2.863 & 1.196 & -0.240 \\
\hline 2,6-difluoropyridine & 2.259 & 1.244 & -0.190 & $\ldots$ & $\ldots$ & $\ldots$ & 2.199 & 1.246 & -0.188 \\
\hline 3,4-difluoropyridine & $3.025^{\mathrm{b}}$ & 1.172 & -0.268 & $\ldots$ & $\ldots$ & $\ldots$ & 3.024 & 1.173 & -0.267 \\
\hline 3,5-difluoropyridine & 3.519 & 1.148 & -0.291 & $\ldots$ & $\ldots$ & $\ldots$ & 3.527 & 1.146 & -0.292 \\
\hline Pentafluoropyridine & 1.970 & 1.257 & -0.163 & $\ldots$ & $\ldots$ & $\ldots$ & 1.775 & 1.252 & -0.169 \\
\hline
\end{tabular}

${ }^{\mathrm{a}}$ Contribution from fluorine in the 2-, 3-, or 4- position to $\chi_{\mathrm{cc}}, 2 p_{\mathrm{z}}$ or $-\left(U_{\mathrm{p}}\right)_{\mathrm{z}}$.

${ }^{\mathrm{b}}$ No experimental value available, only theoretical.

${ }^{c}$ Value calculated from that of non-substituted pyridine and the corresponding contributions from monofluoropyridines.

The $e Q q_{210}$ values obtained are $-10.76(10) \mathrm{MHz},-10.97(11)$ $\mathrm{MHz}$, and $-10.85(14) \mathrm{MHz}$ with an $\mathrm{r}^{2}$ of $0.991,0.990$, and 0.984 using B3LYP/cc-pVDZ, B3PW91/6-311+G(df,pd), and MP2/cc$\mathrm{pVDZ}$, respectively. The average from the three levels of theory will be $e Q q_{210}=-10.86(11) \mathrm{MHz}$, which is within the range given previously ( $-10 \mathrm{MHz}$ to $-11 \mathrm{MHz})$, but with higher accuracy in its determination and with the negative sign as suggested by most of the contemporary authors. ${ }^{3,8}$ To try to reduce the uncertainty, we have also performed the fit separating the dataset in two groups; monomers and complexes, since the latter could, in principle, present a higher

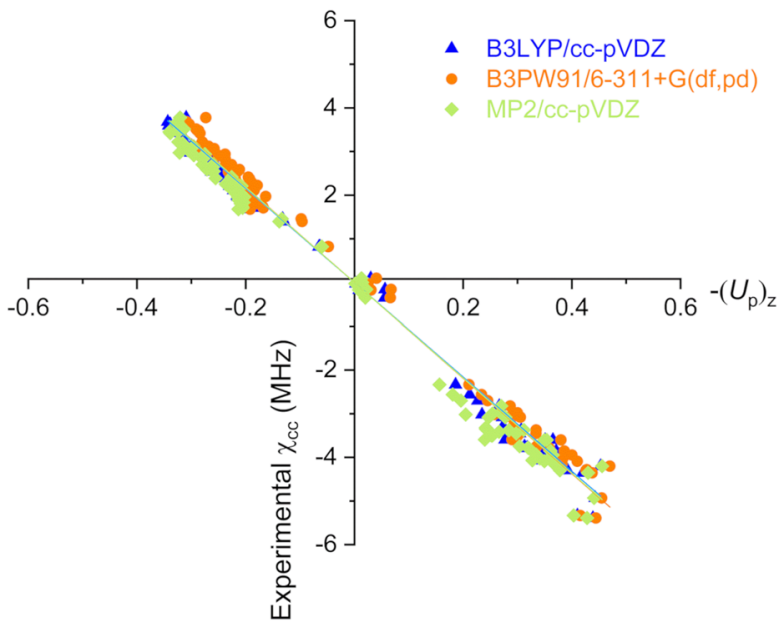

FIG. 7. Experimental $\chi_{c c}$ vs calculated $-\left(U_{p}\right)_{z}$ at the B3LYP/cc-pVDZ, B3PW91/6$311+G(d f, p d)$, and MP2/cc-pVDZ levels of theory. The gradient of the regression line corresponds to $e Q q_{210}$. deviation based on their higher $P_{\mathrm{cc}}$ values (Fig. S5). The result of this fit is comparable to the one including every molecular system, indicating that although complexation may affect the planarity of the system, the approximation $\chi_{\mathrm{cc}} \approx \chi_{\mathrm{zz}}=-\left(U_{\mathrm{p}}\right)_{\mathrm{z}} \cdot e Q q_{210}$ is still valid.

\section{CONCLUSIONS}

In this work, we provide a methodology to extract information about nuclear and molecular properties from the experimental NQCC $\chi_{\mathrm{cc}}$ values together with some straightforward quantum-chemical calculations, with the only requirement that the molecular system is planar or near-planar. $\chi_{\mathrm{cc}}$ can be determined with high accuracy in a straightforward manner from highresolution FTMW spectroscopy. With the methodology described in Secs. II and III, it is possible to establish a relationship between $\chi_{\mathrm{cc}}$ and the unbalanced $p_{z}$ electronic charge. This methodology, first used for formamide/water complexes, ${ }^{22,23}$ was applied to a plethora of molecular systems containing ${ }^{14} \mathrm{~N}$. The values and tendencies in the NQCC $\chi_{\mathrm{cc}}$ showed polarization processes in a series of complexes and allowed tracing out the electron density in substituted aromatic rings. The most outstanding conclusion from this work is the derivation of an accurate semi-experimental value for $e Q q_{210}$ for ${ }^{14} \mathrm{~N}$ from a simple linear fit. The exact value of this parameter was still an open question for physical chemistry. The use of an extensive dataset and the comparison between several levels of theory contribute to a decreased uncertainty in the determination of $e Q q$. The derived value of $e Q q_{210}=-10.86(11) \mathrm{MHz}$ could be used instead of the preceding accepted value of $-11.2 \mathrm{MHz}$ when a higher accuracy in the nuclear quadrupole coupling constants is desired. A more accurate value may yet be obtained once a rigorous benchmarking of DFT, MP2, or coupled cluster methods has determined which combination of method and basis set most accurately predicts the electric field gradient at a nitrogen nucleus. Our general methodology, based 
on the comparison of experimental $\chi_{\mathrm{cc}}$ values obtained from a bibliographical search and simple quantum-chemical calculations using low-cost levels of theory, can be easily extended to other quadrupolar nuclei to obtain their correspondent $e Q q_{\mathrm{n} 10}$ values.

\section{SUPPLEMENTARY MATERIAL}

The supplementary material includes experimental values for the molecular systems included in this work and calculated parameters at the three levels of theory. Resonance structures and additional plots are also included. The complete list of references from which values for this work have been taken is provided in the supplementary material.

\section{ACKNOWLEDGMENTS}

We would like to thank Professor S. Novick. This work was inspired by him, and he has continuously supported us to publish it. A considerable amount of the data points was found at Professor W. C. Bailey's website; we would like to thank him for building and maintaining such a useful site. We acknowledge fruitful discussions with Dr. W. Sun. P.P. is grateful for a postdoctoral fellowship from the Alexander von Humboldt Foundation.

\section{DATA AVAILABILITY}

The data that support the findings of this study are available within the article and its supplementary material and from the authors upon reasonable request.

\section{REFERENCES}

${ }^{1}$ C. H. Townes and B. P. Dailey, J. Chem. Phys. 17, 782 (1949).

${ }^{2}$ C. H. Townes and A. L. Schawlow, Microwave Spectroscopy (McGraw-Hill Publishing Company, New York, 1955).

${ }^{3}$ W. Gordy and R. L. Cook, Microwave Molecular Spectra (Wiley-Interscience, New York, 1972).

${ }^{4}$ B. P. Dailey and C. H. Townes, J. Chem. Phys. 23, 118 (1955).

${ }^{5}$ E. R. Cohen, T. Cvitas, J. G. Frey, B. Holmstrom, K. Kuchitsu, R. Marquardt, I. Mills, F. Pavese, M. Quack, J. Stohner, H. Strauss, M. Takami, and A. J. Thor, Quantities, Units, and Symbols in Physical Chemistry, IUPAC Green Book, 3rd ed. (RSC Publishing, 2007).

${ }^{6}$ K. Gaul and R. Berger, Mol. Phys. 118, e1797199 (2020).

${ }^{7}$ P. Schwerdtfeger, R. Bast, M. C. L. Gerry, C. R. Jacob, M. Jansen, V. Kellö, A. V. Mudring, A. J. Sadlej, T. Saue, T. Söhnel, and F. E. Wagner, J. Chem. Phys. 122, 124317 (2005)

${ }^{8}$ S. E. Novick, J. Mol. Spectrosc. 267, 13 (2011).

${ }^{9}$ Y. Kato, U. Furukane, and H. Takeyama, Bull. Chem. Soc. Jpn. 32, 527 (1959).

${ }^{10}$ S. S. Lehrer and C. T. O’Konski, J. Chem. Phys. 43, 1941 (1965).

${ }^{11}$ C. T. O. O’Konski and T.-K. Ha, J. Chem. Phys. 56, 3169 (1972).
${ }^{12}$ A. Schirmacher and H. Winter, Phys. Rev. A 47, 4891 (1993).

${ }^{13}$ A. Abragam, The Principles of Nuclear Magnetism (Oxford University Press, Oxford, 1961).

${ }^{14}$ D. D. Laws, H.-M. L. Bitter, and A. Jerschow, Angew. Chem., Int. Ed. 41, 3096 (2002).

${ }^{15}$ D. L. Bryce and R. E. Wasylishen, Acc. Chem. Res. 36, 327 (2003).

${ }^{16}$ S. E. Ashbrook and M. J. Duer, Concepts Magn. Reson., Part A: Bridging Educ. Res. 28A, 183 (2006).

${ }^{17}$ A. E. Reed, R. B. Weinstock, and F. Weinhold, J. Chem. Phys. 83, 735 (1985).

${ }^{18}$ A. E. Reed, L. A. Curtiss, and F. Weinhold, Chem. Rev. 88, 899 (1988).

${ }^{19}$ A. Rinald and G. Wu, J. Phys. Chem. A 124, 1176 (2020).

${ }^{20}$ S. Blanco, A. Lesarri, J. C. López, and J. L. Alonso, J. Am. Chem. Soc. 126, 11675 (2004).

${ }^{21}$ J. L. Alonso, V. Cortijo, S. Mata, C. Pérez, C. Cabezas, J. C. López, and W. Caminati, J. Mol. Spectrosc. 269, 41 (2011).

${ }^{22}$ S. Blanco, P. Pinacho, and J. C. López, Angew. Chem., Int. Ed. 128, 9477 (2016).

${ }^{23}$ S. Blanco, P. Pinacho, and J. C. López, J. Phys. Chem. Lett. 8, 6060 (2017).

${ }^{24} \mathrm{D}$. Loru et al. (unpublished).

${ }^{25}$ T. J. Balle and W. H. Flygare, Rev. Sci. Instrum. 52, 33 (1981); U. Andresen, H. Dreizler, J. U. Grabow, and W. Stahl, ibid. 61, 3694 (1990).

${ }^{26}$ G. G. Brown, B. C. Dian, K. O. Douglass, S. M. Geyer, and B. H. Pate, J. Mol. Spectrosc. 238, 200 (2006); G. G. Brown, B. C. Dian, K. O. Douglass, S. M. Geyer, S. T. Shipman, and B. H. Pate, Rev. Sci. Instrum. 79, 053103 (2008).

${ }^{27}$ W. B. Bailey, "Calculation of nuclear quadrupole coupling contants in gaseous state molecules," available at http://nqcc.wcbailey.net/.

${ }^{28}$ C. Lee, W. Yang, and R. G. Parr, Phys. Rev. B 37, 785 (1988); A. D. Becke, J. Chem. Phys. 98, 5648 (1993); S. H. Vosko, L. Wilk, and M. Nusair, Can. J. Phys. 58, 1200 (1980).

${ }^{29}$ T. H. Dunning, J. Chem. Phys. 90, 1007 (1989).

${ }^{30}$ J. P. Perdew, P. Ziesche, and H. Eschrig, Electronic Structure of Solids'91 (Akademie Verlag, Berlin, 1991).

${ }^{31}$ K. Raghavachari, J. S. Binkley, R. Seeger, and J. A. Pople, J. Chem. Phys. 72, 650 (1980).

${ }^{32}$ C. Møller and M. S. Plesset, Phys. Rev. 46, 618 (1934).

${ }^{33}$ S. F. Sousa, P. A. Fernandes, and M. J. Ramos, J. Phys. Chem. A 111, 10439 (2007).

${ }^{34}$ W. C. Bailey, Chem. Phys. 252, 57 (2000).

${ }^{35}$ M. J. Frisch et al., Gaussian 09, Revision A.02, Gaussian, Inc., Wallingford, CT, 2009.

${ }^{36}$ S. Blanco, J. C. López, A. Lesarri, and J. L. Alonso, J. Am. Chem. Soc. 128, 12111 (2006).

${ }^{37}$ L. Nygaard, I. Bojesen, T. Pedersen, and J. Rastrup-Andersen, J. Mol. Struct. 2, 209 (1968).

${ }^{38}$ S. Doraiswamy and S. D. Sharma, J. Mol. Struct. 102, 81 (1983).

${ }^{39}$ C. W. Van Dijk, M. Sun, and J. van Wijngaarden, J. Phys. Chem. A 116, 4082 (2012).

${ }^{40}$ C. W. Van Dijk, M. Sun, and J. van Wijngaarden, J. Mol. Spectrosc. 280, 34 (2012).

${ }^{41}$ C. Calabrese, Q. Gou, L. Spada, A. Maris, W. Caminati, and S. Melandri, J. Phys. Chem. A 120, 5163 (2016).

${ }^{42}$ J. B. Graneek, W. C. Bailey, and M. Schnell, Phys. Chem. Chem. Phys. 20, 22210 (2018).

${ }^{43}$ A. Y. Chung, E. A. Arsenault, S. L. Stephens, W. C. Pringle, C. A. JiménezHoyos, S. A. Cooke, and S. E. Novick, J. Mol. Spectrosc. 356, 28 (2019). 